\title{
Management and Outcome of Ventricular Septal Rupture Complicating Acute Myocardial Infarction: What Is New in the Era of Percutaneous Intervention?
}

\author{
Shi Tai ${ }^{a} \quad$ Jian-jun Tang ${ }^{a}$ Liang Tang ${ }^{a} \quad$ Yu-qing Nia Yanan Guo ${ }^{a}$ Xin-qun $\mathrm{Hu}^{\mathrm{a}}$ \\ Zhen-fei Fang $^{\mathrm{a}}$ Hong-wei Pan $^{\mathrm{b}}$ He Huang ${ }^{c}$ Chang-hui Liu ${ }^{d}$ Gao-feng Zeng ${ }^{\mathrm{e}}$ \\ Sheng-hua Zhou ${ }^{\mathrm{a}}$ \\ a Department of Cardiology, The Second Xiangya Hospital of Central South University, Changsha, China; \\ ${ }^{b}$ Department of Cardiology, Hunan Provincial People's Hospital, Changsha, China; ' Department of Cardiology, \\ Xiangtan Central Hospital, Xiangtan, China; ${ }^{\mathrm{d} D e p a r t m e n t}$ of Cardiology, The First Affiliated Hospital of University of

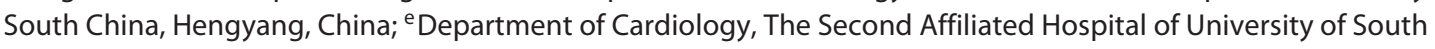 \\ China, Hengyang, China
}

\section{Keywords}

Ventricular septal rupture - Acute myocardial infarction · Risk factors · Mechanical circulatory support · Percutaneous device closure

\begin{abstract}
Background: Postinfarction ventricular septal rupture (PIVSR) is a rare but devastating complication of acute myocardial infarction (AMI). Risk stratification in the acute phase is crucial for decision-making, and this study analyzed the risk factors for early mortality and the effects of various management options on the outcome of PI-VSR patients in the era of percutaneous intervention. Methods: A total of 96 patients with PI-VSR were identified and divided into an acute-phase survivor group ( $n=46$, survived $\geq 2$ weeks after admission) and a nonsurvivor group ( $n=50$, died within 2 weeks after admission). Percutaneous closure was considered in acutephase survivors. Patients were followed up for a mean 47 (quartiles 15-71) months by clinical visit or telephone interview. Results: The overall acute-phase (i.e., $<2$ weeks after
\end{abstract}

the diagnosis of PI-VSR) mortality rate was 52\%. Female sex and Killip Class III-IV at admission were associated with an increased risk of acute-phase death. Of the 46 patients who survived $\geq 2$ weeks, 20 underwent interventional occlusion and the procedure was successful in 19. Percutaneous closure in the acute-phase survivor group improved the immediate (21\% in-hospital mortality rate) and long-term (53\% mortality) outcomes. Conclusions: Patients with PI-VSR are at a high risk of acute-phase mortality. Female sex and severe cardiac dysfunction at admission are linked with a high rate of acute-phase deaths. Percutaneous closure in acute-phase survivors results in favorable short- and long-term benefits for PI-VSR patients.

๑) 2019 S. Karger AG, Basel

\section{Introduction}

Postinfarction ventricular septal rupture (PI-VSR) is a rare but life-threatening mechanical complication of acute myocardial infarction (AMI) [1]. While the inci-

\section{KARGER}

(c) 2019 S. Karger AG, Basel

E-Mail karger@karger.com

www.karger.com/crd
Sheng-hua Zhou, MD, $\mathrm{PhD}$

Professor of Cardiology and Director of Department of Cardiology The Second Xiangya Hospital of Central South University

No.139, Middle Ren-Min Road, Changsha, Hunan 410011 (PR China)

E-Mail zhoushenghua@ csu.edu.cn 
dence of PI-VSR has decreased in the era of reperfusion, the mortality rate remains extremely high $[2,3]$.

Promising outcomes have been reported with the use of transcatheter closure of PI-VSR, allowing immediate shunt reduction to prevent hemodynamic deterioration [4]. Hence, transcatheter closure has become a valuable alternative to surgical repair, with a high rate of procedural success [5]. Nevertheless, an important heterogeneity concerning the timing of transcatheter closure can be observed among studies [5-10]. The current ST-elevation myocardial infarction (STEMI) guidelines of the European Society of Cardiology (ESC) and the American College of Cardiology Foundation/American Heart Association (ACCF/AHA) recommend urgent PI-VSR closure irrespective of hemodynamic status $[11,12]$, but the timing of PI-VSR closure and perioperative therapeutic management remain controversial [13]. In previous studies, most percutaneous device closures were performed during the subacute and chronic periods ( $\geq 2$ weeks) after the initial detection of PI-VSR $[4,8,10,14]$. The mortality associated with delayed percutaneous closure ( $\geq 2$ weeks after PI-VSR detection) was reported to be $6.1-10.0 \%$ [8, $10]$, while mortality after early closure $(<2$ weeks after PIVSR occurrence) was as high as $66 \%$ [6]. This is likely explained by a selection bias, so the patients considered too sick to wait for delayed closure and those who died during the waiting period need to be identified.

Risk stratification is essential to improve the decisionmaking for PI-VSR patients. The goals of this study were: (1) to compare the clinical and angiographic features of PI-VSR patients who died or survived within 2 weeks after identification of PI-VSR and identify the risk factors for early (i.e., <2 weeks) mortality of PI-VSR patients; (2) to evaluate the effects of management options including PI-VSR closure in acute-phase survivors and identify risk factors related to the mortality of these patients after PI-VSR closure.

\section{Methods}

\section{Patient Selection}

Our retrospective analysis included 96 patients with PI-VSR hospitalized at 5 Chinese heart centers from December 2007 to July 2017. Primary data were extracted from the electronic medical or archived records by trained staff. Data were collected regarding patient demographics, clinical features, preprocedural clinical condition, echocardiography features, and procedural variables as well as treatment options (including medical and surgical), and outcomes of all patients. The inclusion criteria were: (1) patients admitted for AMI and had emergent cardiac catheterization for VSR; (2) AMI patients with hemodynamic compromise with echo- cardiographic evidence of VSR. Patients who died at initial presentation were excluded, including those who did not survive the emergent cardiac catheterization. Diagnosis of AMI was made according to the definition of myocardial infarction from the Fourth Universal Definition of Myocardial Infarction [15].

\section{Coronary Angiography and Stenting}

Coronary angiography was performed using the standard percutaneous radial artery approach. The femoral approach was used when an intra-aortic balloon pump (IABP) was required in case of hemodynamic instability. Percutaneous closure was performed immediately if hemodynamics continually worsened after using IABP. All angiographic data obtained from the catheterization laboratory records were assessed using conventional protocols. Of the 20 patients with VSR interventional occlusion attempts, 17 did not undergo percutaneous intervention (PCI) before VSR occlusion because they exceeded the time window for performing emergency PCI. The timing of PCI and antiplatelet therapy was at the physicians' discretion. Usually, VSD closure was performed prior to PCI, but PCI would proceed closure if the patient presented with unstable angina and if coronary angiography showed a culprit lesion in proximal vessels with a heavy thrombosis burden [8]. All patients received standardized treatment for AMI $[11,12]$; the PCI strategy and antiplatelet therapy were performed at the discretion of the physician.

\section{Device and Interventional Closure}

The closure devices and implantation technique were similar to those described in our previous report [16]. Vascular access was generally obtained via the right femoral artery and vein, but the femoral arterial and jugular venous approach was used for arteriovenous guidewire-loop creation in patients with an apical VSR. All patients had a chest X-ray, transthoracic echocardiography, and electrocardiogram within $24 \mathrm{~h}$ following the procedure. All patients completed a median clinical follow-up of 47 (quartiles 1571) months in the form of a clinical visit or telephone interview.

\section{Statistical Analysis}

Statistical analysis was performed using SPSS v23.0 (IBM, Armonk, NY, USA). All continuous variables were expressed as mean \pm standard deviation (SD) or median (range), as appropriate. Categorical data were presented as frequency (percentage). Comparisons of baseline data were performed using the $\chi^{2}$ test or Fisher's exact test (categorical variables) and Student's $t$ test or the Wilcoxon rank sum test, as appropriate (continuous variables). Clinical and procedural parameters were tested for an association with acute-phase death using logistic regression. All tests were two-sided and $p<0.05$ was considered statistically significant.

\section{Results}

\section{Patient and Clinical Characteristics}

Between December 2007 and July 2017, 96 patients were diagnosed with PI-VSR and met the inclusion criteria for this study. Baseline demographic and clinical data of these patients are listed in Table 1. The patients were $66.0 \pm 10.7$ years old and $46(48 \%)$ were female. Patients 
Table 1. Demographic data and clinical characteristics

\begin{tabular}{lcccc}
\hline & $\begin{array}{l}\text { All } \\
(n=96)\end{array}$ & $\begin{array}{l}\text { Acute-phase } \\
\text { nonsurvivors } \\
(n=50)\end{array}$ & $\begin{array}{l}\text { Acute-phase } \\
\text { survivors } \\
(n=46)\end{array}$ & $p$ value \\
\hline Age, years & $66 \pm 10.70$ & $68 \pm 11.00$ & $63 \pm 9.77$ & 0.016 \\
Female sex & $41(42.70)$ & $27(54)$ & $14(30)$ & 0.020 \\
Diabetes mellitus & $25(26.04)$ & $12(24)$ & $13(28)$ & 0.635 \\
Hypertension & $58(60.41)$ & $32(64)$ & $26(57)$ & 0.454 \\
Smoking & $37(38.54)$ & $15(30)$ & $22(48)$ & 0.073 \\
Hypercholesterolemia & $22(22.91)$ & $10(20)$ & $12(26)$ & 0.478 \\
History of angina & $19(19.79)$ & $6(12)$ & $13(28.3)$ & 0.046 \\
Killip Class & $31(32.29)$ & $10(20)$ & $21(46$ & 0.012 \\
$\quad$ I-II & $65(67.71)$ & $41(82)$ & $24(52)$ & 0.002 \\
$\quad$ III-IV & $51.74 \pm 8.15$ & $48.19 \pm 7.47$ & $54.05 \pm 7.81$ & 0.004 \\
LVEDV, mm & $50.92 \pm 12.20$ & $50.87 \pm 11.27$ & $50.95 \pm 12.91$ & 0.980 \\
LVEF, $\%$ & $31(32.29)$ & $18(36)$ & $13(28)$ & 0.051 \\
Infarct territory & $52(54.17)$ & $28(56)$ & $24(52)$ & 0.202 \\
$\quad$ Inferior & $13(13.54)$ & $6(12)$ & $7(15)$ & 0.476 \\
$\quad$ Anterior & $144.55 \pm 82.18$ & $163.09 \pm 88.18$ & $124.40 \pm 70.65$ & 0.020 \\
$\quad$ Others & $49(51.04)$ & $18(38)$ & $31(67)$ & 0.002 \\
Creatinine, $\mu$ mol/L & $60(63)$ & $32(64)$ & $28(61)$ & 0.752 \\
Ventricular aneurysm & & & & \\
IABP & & & & \\
\hline
\end{tabular}

Values are expressed as $n(\%)$ or mean \pm SD. LVEDV, left ventricular end-diastolic volume; LVEF, left ventricular ejection fraction; IABP, intra-aortic balloon pump.

with PI-VSR were grouped as acute-phase survivors $(n=$ 46 , survived $\geq 2$ weeks after diagnosis of PI-VSR) and nonsurvivors $(n=50$, died within 2 weeks after the diagnosis of PI-VSR). Patients who developed VSR and died within 2 weeks were more likely to be older $(p=0.016)$, female ( $p=0.020)$, and more often had Killip Class III-IV at admission $(p=0.002)$ than those who survived $\geq 2$ weeks (Table 1). Meanwhile, patients in the acute-phase survivor group more often had a history of angina or myocardial infarction $(p=0.046)$ or ventricular aneurysm $(p=0.002)$, and a larger left ventricular end-diastolic volume (LVEDV) $(p=0.004)$ (Table 1). Logistic regression analysis revealed that female sex (odds ratio [OR] 5.651; 95\% confidence interval [CI] 1.095-29.150; $p=$ 0.039 ) and Killip Class III-IV at admission (OR 5.487; 95\% CI 1.211-24.863; $p=0.027$ ) were risk factors for acute-phase death after VSR occurrence (Table 2). On the other hand, a history of angina and ventricular aneurysm were protective factors against acute-phase death in PIVSR patients.

This study observed that $82 \%$ of AMI patients with VSR and Killip class III-IV at admission died within 2 weeks after a diagnosis of PI-VSR. The territory of the in- farction was anterior in 52 patients (54\%) and inferior in $31(32 \%)$; there was no significant difference between the 2 groups. The use of IABP was common in both groups.

\section{Angiographic Characteristics}

Angiographic data were available for 62 of the 96 PIVSR patients (65\%) (Table 3). Patients with PI-VSR were more likely to have TIMI grade 0 or 1 flow at first coronary angiography, and $60 \%$ had total occlusion of the infarct artery. The infarct-related artery was more often the LAD in patients with PI-VSR. Forty-five out of the 62 patients (73\%) had 2- or 3-vessel disease. The PI-VSR patients who died within 2 weeks after diagnosis were more likely to have a culprit lesion in the LAD and a lower incidence of collateral flow to the infarcted area than those who survived $\geq 2$ weeks after diagnosis. Nevertheless, LAD infarct and absence of collateral flow were not independent predictors of mortality.

\section{Percutaneous Device Closure and Follow-Up Results}

PI-VSR was considered for acute-phase survivors. Closure was unsuitable for 26 patients due to instable clinical conditions, so they received conservative therapy; 
Table 2. Clinical factors associated with early mortality

\begin{tabular}{lllllll}
\hline Risk factors & \multicolumn{1}{l}{ B } & SE & OR & $95 \%$ CI & & $p$ value \\
\hline Female sex & 1.732 & 0.837 & 5.651 & 1.095 & 29.150 & 0.039 \\
A history of angina & -3.276 & 1.102 & 0.038 & 0.004 & 0.327 & 0.003 \\
Killip Class III-IV & 1.702 & 0.771 & 5.487 & 1.211 & 24.863 & 0.027 \\
Ventricular aneurysm & -1.834 & 0.701 & 0.160 & 0.040 & 0.631 & 0.009
\end{tabular}

Table 3. Angiographic findings in patients with PI-VSR

\begin{tabular}{lllll}
\hline Coronary angiography & $\begin{array}{l}\text { All } \\
(n=62)\end{array}$ & $\begin{array}{l}\text { Acute-phase } \\
\text { nonsurvivors }(n=23)\end{array}$ & $\begin{array}{l}\text { Acute-phase } \\
\text { survivors }(n=39)\end{array}$ & $p$ value \\
\hline Infarct-related artery & & & & $25(64 \%)$ \\
$\quad$ LAD & 44 & $19(82 \%)$ & $13(33 \%)$ & 0.121 \\
RCA & 18 & $5(22 \%)$ & $9(23 \%)$ & 0.207 \\
Single vessels & 17 & $8(35 \%)$ & $30(77 \%)$ & 0.521 \\
Multiple vessels & 45 & $15(65 \%)$ & $8(21 \%)$ & 0.222 \\
Collateral circulation & 10 & $2(9 \%)$ & &
\end{tabular}

LAD, left anterior descending artery; RCA, right coronary artery.

they all died during hospitalization due to cardiogenic shock. Twenty of 46 patients in the acute-phase survivor group received percutaneous closure. The median time from PI-VSR diagnosis to percutaneous closure was 23 (range 14-62) days. The infarction territory was anterior in 8 patients (40\%) and inferior in $12(60 \%)$. The defect site was evenly distributed between anterior $(n=5)$, apical $(n=9)$, and inferior territories $(n=6)$. Devices were successfully implanted in 19 patients (95\%). The reason for failed closure in 1 patient was the presence of a diffuse defect; this patient received emergent surgical repair after failed percutaneous closure but died hours later due to refractory cardiac shock. In the 19 patients with successful percutaneous closure, the median VSR size was 13 (range $8-21$ ) $\mathrm{mm}$ and the median device size was 19 (range 14-32) mm. In total, 4 patients died within 0-7 days after the successful percutaneous closure (in-hospital mortality, 20\%). Reasons for early death included: LV free wall rupture $(n=1)$, cerebral hemorrhage $(n=1)$, and persistent shock and multiple organ failures $(n=2)$. Fifteen patients were discharged and received regular clinical or telephone follow-up for a median 47 (quartiles 1571) months. Seven patients were still alive. Clinical features of survivors and nonsurvivors after percutaneous closure are listed in Table 4. Although the number of PIVSR patients who underwent percutaneous device clo- sure was insufficient to fully determine the characteristics associated with long-term mortality, there was a tendency suggesting that younger age and smaller defect size are favorable factors for long-term survival in this setting.

\section{Discussion}

PI-VSR is an infrequent but devastating complication of AMI. With advances in interventional technologies and experience, the management of this complication has improved. This study demonstrated that $52 \%$ of all patients died within the first 2 weeks after PI-VSR, even with optimal medical treatment and/or IABP support. The patients who survived $\geq 2$ weeks and underwent successful percutaneous closure showed a trend toward a favorable long-term outcome.

Risk Factors for Early Death in Patients with PI-VSR

Patients who developed VSR and died within 2 weeks were more likely to be of advanced age and female than those who survived $\geq 2$ weeks. In the SHOCK and GUSTO-I studies $[1,17]$, these factors were also associated with PI-VSR occurrence. Furthermore, advanced age and female sex were identified as risk factors for mortality in a long-term follow-up of PI-VSR patients [9]. 
Table 4. Follow-up results for patients after percutaneous closure

\begin{tabular}{|c|c|c|c|c|}
\hline & $\begin{array}{l}\text { All } \\
(n=15)\end{array}$ & $\begin{array}{l}\text { Survivors } \\
(n=7)\end{array}$ & $\begin{array}{l}\text { Nonsurvivors } \\
(n=8)\end{array}$ & $p$ value \\
\hline Mean age, years $( \pm S D)$ & $63 \pm 9.845$ & $60 \pm 12.075$ & $65 \pm 7.305$ & 0.307 \\
\hline Female sex, $n$ & 9 & $3(33 \%)$ & $6(67 \%)$ & 0.315 \\
\hline Diabetes mellitus, $n$ & 7 & $4(57 \%)$ & $3(43 \%)$ & 0.619 \\
\hline Hypertension, $n$ & 8 & $5(62.5 \%)$ & $3(37.5 \%)$ & 0.315 \\
\hline Smoking, $n$ & 7 & $4(57 \%)$ & $3(43 \%)$ & 0.619 \\
\hline Hypercholesterolemia, $n$ & 4 & $1(25 \%)$ & $3(75 \%)$ & 0.569 \\
\hline \multicolumn{5}{|l|}{ Killip class, $n$} \\
\hline I-II & 6 & $3(50 \%)$ & $3(50 \%)$ & \multirow[t]{2}{*}{1.00} \\
\hline III-IV & 9 & $4(44 \%)$ & $5(56 \%)$ & \\
\hline Mean LVEDV, mm $( \pm$ SD) & $51.8 \pm 6.247$ & $53.43 \pm 4.93$ & $50.38 \pm 7.23$ & 0.364 \\
\hline Mean LVEF, \% ( \pm SD) & $48.31 \pm 6.421$ & $49.5 \pm 7.06$ & $47.29 \pm 6.184$ & 0.559 \\
\hline \multicolumn{5}{|l|}{ Infarct territory, $n$} \\
\hline Inferior & 6 & $4(66 \%)$ & $2(34 \%)$ & \multirow[t]{2}{*}{0.315} \\
\hline Anterior & 9 & $3(33 \%)$ & $6(67 \%)$ & \\
\hline Mean creatinine, $\mu \mathrm{mol} / \mathrm{L}( \pm \mathrm{SD})$ & $115 \pm 37.97$ & $125 \pm 19.18$ & $107 \pm 49.03$ & 0.391 \\
\hline Median VSD to procedure time, days (quartiles $1-3$ ) & $23(20-29)$ & $20(17-24)$ & $24(22-30)$ & 0.232 \\
\hline \multicolumn{5}{|l|}{ Site of defect, $n$} \\
\hline Anterior & 4 & $1(25 \%)$ & $3(75 \%)$ & \multirow[t]{3}{*}{0.566} \\
\hline Apical & 5 & $3(60 \%)$ & $2(40 \%)$ & \\
\hline Inferior & 6 & $3(50 \%)$ & $3(50 \%)$ & \\
\hline Median size of defect, mm (quartiles 1-3) & $13(7-20)$ & $10(6-19)$ & $15(12-15)$ & 0.629 \\
\hline \multicolumn{5}{|l|}{ Type of percutaneous closure performed, $n$} \\
\hline VSD & 11 & $5(45 \%)$ & $6(55 \%)$ & \multirow[t]{3}{*}{0.506} \\
\hline ASD & 3 & $1(33 \%)$ & $2(67 \%)$ & \\
\hline PDA & 1 & $1(100 \%)$ & $0(0 \%)$ & \\
\hline
\end{tabular}

LVEDV, left ventricular end-diastolic volume; LVEF, left ventricular ejection fraction; VSD, ventricular septal defect; ASD, atrial septal defect; PDA, patent ductus arteriosus.

Cardiac shock in PI-VSR patients has been associated with an increased risk of death $[7,9,17,18]$. Similarly, our study showed that the majority of PI-VSR patients who had Killip class III-IV at admission died within 2 weeks. These findings further underline the importance of stabilizing the hemodynamics at an early stage of PI-VSR. Interestingly, acute-phase survivors of PI-VSR often experienced complications like ventricular aneurysm and a larger LVEDV. It can be hypothesized that the formation of ventricular aneurysm and a larger LVEDV contribute to the decreased pressure gradient between the left and right ventricles, which prevents further tearing of the septal tissue.

The prevalence of a history of angina or myocardial infarction was also higher in the acute-phase survivors of PI-VSR. This finding hints at a likelihood of more coronary collateral formation in these patients. In fact, angiographic examination confirmed that PI-VSR patients who died within 2 weeks after the diagnosis of PI-VSR were less likely to have collateral flow to the infarcted area and more likely to have a culprit lesion in the LAD than those who survived for $\geq 2$ weeks.

\section{Timing of Percutaneous Closure}

The optimal therapeutic strategy and timing for VSR closure in patients with PI-VSR is a matter of ongoing debate. Although current guidelines recommend immediate intervention in patients with PI-VSR regardless of their clinical status $[11,12]$, the majority of percutaneous closures were performed during the subacute or chronic phase $[4,8,10]$. In our series, among the 19 acute-phase survivors who had relatively stable hemodynamics and underwent successful closure, only 4 (21\%) died before discharge. In a prospective analysis, Zhu et al. [8] reported a mortality of only $10 \%$ in 22 PI-VSR patients post percutaneous closure during the subacute or chronic phase. Similarly, Xu et al. [10] reported a 97\% procedure success rate in $33 \mathrm{PI}-\mathrm{VSR}$ patients at subacute or chronic phase. These results collectively suggest that delayed percutaneous closure of PI-VSR might be feasible and effec- 
tive for subacute- or chronic-phase PI-VSR patients. However, the overall 30-day in-hospital mortality rate after acute percutaneous closure remained high. Zhu et al. [8] and Xu et al. [10] reported 23 and $66.7 \%$ mortality in patients who underwent percutaneous closure within the first 2 weeks after PI-VSR occurrence. Furthermore, Thiele et al. [6] reported a mortality rate of $66 \%$ in PI-VSR patients who underwent primary percutaneous closure in the acute phase. Similarly, high mortality was observed in early surgical series, while operative mortality was reduced in PI-VSR patients receiving delayed surgical repair [19]. The reason for high mortality in patients undergoing percutaneous or surgical repair in the acute phase might relate to the infarcted myocardium, which is weak and friable in the acute phase following AMI and, together with the unstable hemodynamic status, serves as an unfavorable factor for the high mortality of PI-VSR patients associated with repair conducted in the acute phase.

\section{Limitations}

The main limitation of this study was the retrospective design. Due to the low incidence of PI-VSR in the current era of thrombolytic and interventional treatment of AMI, the study population was relatively small and the study period spanned a long time. In addition, the study lacked randomized comparisons between acute-phase and delayed transcatheter therapy. However, the relevant literature was reviewed in order to assess the best approach for this population.

\section{Conclusion}

Our study confirms the high acute-phase mortality of patients with PI-VSR. Female sex and Killip Class III-IV at admission were associated with an increased risk of death in the acute phase ( $<2$ weeks after the diagnosis of PI-VSR). Most importantly, our data revealed the satis- factory outcomes of patients who survived the acute phase and underwent successful percutaneous closure, the in-hospital mortality was low (21\%), and around half of the patients discharged from the hospital were still alive at a median of 47 (quartiles 15-71) months of follow-up.

\section{Acknowledgements}

We gratefully acknowledge the help of Dr. Sybille Redmond in the Minneapolis Heart Institute Foundation.

\section{Statement of Ethics}

The study was approved by the Ethics Committee of each participating hospital and was carried out in accordance with the Declaration of Helsinki.

\section{Disclosure Statement}

There were no conflicts of interest.

\section{Funding Sources}

This research was supported by the Natural Science Foundation of China (81670269 to S-h.Z.).

\section{Author Contributions}

The work presented here was carried out by collaboration of all authors. Sheng-hua Zhou and Jian-jun Tang defined the study theme and methods. Shi Tai, Liang Tang, Yanan Guo, and Yu-qing $\mathrm{Ni}$ collected and analyzed the data, interpreted the results, and wrote the paper. Xin-qun $\mathrm{Hu}$, Zhen-fei Fang, Hong-wei Pan, $\mathrm{He}$ Huang, Chang-hui Liu, and Gao-feng Zeng were the attending doctors responsible for the treatment of patients. All authors contributed to and approved the manuscript.

\section{References}

1 Crenshaw BS, Granger CB, Birnbaum Y, Pieper KS, Morris DC, Kleiman NS, et al; GUSTO-I Trial Investigators. Risk factors, angiographic patterns, and outcomes in patients with ventricular septal defect complicating acute myocardial infarction. Circulation. $2000 \mathrm{Jan} ; 101(1): 27-32$.

2 Moreyra AE, Huang MS, Wilson AC, Deng Y, Cosgrove NM, Kostis JB; MIDAS Study Group (MIDAS 13). Trends in incidence and mortality rates of ventricular septal rupture during acute myocardial infarction. Am J Cardiol. 2010 Oct;106(8):1095-100.

3 Goldsweig AM, Wang Y, Forrest JK, Cleman MW, Minges KE, Mangi AA, et al. Ventricular septal rupture complicating acute myocardial infarction: Incidence, treatment, and outcomes among medicare beneficiaries 19992014. Catheter Cardiovasc Interv. 2018 Nov; 92(6):1104-15.

4 Demkow M, Ruzyllo W, Kepka C, Chmielak Z, Konka M, Dzielinska Z, et al. Primary transcatheter closure of postinfarction ventricular septal defects with the Amplatzer septal occluder- immediate results and up-to 5 years follow-up. EuroIntervention. $2005 \mathrm{May}$; $1(1): 43-7$.

5 Schlotter F, de Waha S, Eitel I, Desch S, Fuernau $G$, Thiele $H$. Interventional post-myocardial infarction ventricular septal defect closure: a systematic review of current evidence. EuroIntervention. 2016 May;12(1): 94-102. 
6 Thiele H, Kaulfersch C, Daehnert I, Schoenauer M, Eitel I, Borger M, et al. Immediate primary transcatheter closure of postinfarction ventricular septal defects. Eur Heart J. 2009 Jan;30(1):81-8.

7 Assenza GE, McElhinney DB, Valente AM, Pearson DD, Volpe M, Martucci G, et al. Transcatheter closure of post-myocardial infarction ventricular septal rupture. Circ Cardiovasc Interv. $2013 \mathrm{Feb}$;6(1):59-67.

8 Zhu XY, Qin YW, Han YL, Zhang DZ, Wang $\mathrm{P}$, Liu YF, et al. Long-term efficacy of transcatheter closure of ventricular septal defect in combination with percutaneous coronary intervention in patients with ventricular septal defect complicating acute myocardial infarction: a multicentre study. EuroIntervention. 2013 Mar;8(11):1270-6.

9 Calvert PA, Cockburn J, Wynne D, Ludman P, Rana BS, Northridge D, et al. Percutaneous closure of postinfarction ventricular septal defect: in-hospital outcomes and long-term follow-up of UK experience. Circulation. 2014 Jun;129(23):2395-402.

10 Xu XD, Liu SX, Liu X, Chen Y, Li L, Qu BM, et al. Percutaneous closure of postinfarct muscular ventricular septal defects: a multicenter study in China. J Cardiol. 2014 Oct; 64(4):285-9.
11 Ibanez B, James S, Agewall S, Antunes MJ, Bucciarelli-Ducci C, Bueno H, et al. 2017 ESC Guidelines for the management of acute myocardial infarction in patients presenting with ST-segment elevation: Task Force for the management of acute myocardial infarction in patients presenting with ST-segment elevation of the European Society of Cardiology (ESC). Eur Heart J. 2018 Jan;39(2):119-77.

12 American College of Emergency Physicians; Society for Cardiovascular Angiography and Interventions, O'Gara PT, Kushner FG, Ascheim DD, Casey DE Jr, Chung MK, de Lemos JA, et al: 2013 ACCF/AHA guideline for the management of ST-elevation myocardial infarction: a report of the American College of Cardiology Foundation/American Heart Association Task Force on Practice Guidelines. J Am Coll Cardiol. 2013 Jan; 61(4):e78-140.

13 Malhotra A, Patel K, Sharma P, Wadhawa V, Madan T, Khandeparkar J, et al. Techniques, Timing \& Prognosis of Post Infarct Ventricular Septal Repair: a Re-look at Old Dogmas. Rev Bras Cir Cardiovasc. 2017 May-Jun; 32(3): $147-55$

14 Holzer R, Balzer D, Amin Z, Ruiz CE, Feinstein J, Bass J, et al. Transcatheter closure of postinfarction ventricular septal defects using the new Amplatzer muscular VSD occluder: results of a U.S. Registry. Catheter Cardiovasc Interv. 2004 Feb;61(2):196-201.
15 Thygesen K, Alpert JS, Jaffe AS, Chaitman BR, Bax JJ, Morrow DA, et al; ESC Scientific Document Group. Fourth universal definition of myocardial infarction (2018). Eur Heart J. 2019 Jan;40(3):237-69.

16 Tang L, Fang Z, Hu X, Tang J, Shen X, Lu X, et al. Non-surgical repair of ventricular septal rupture after acute myocardial infarction. Int J Cardiol. 2015 Apr;185:328-32.

17 Menon V, Webb JG, Hillis LD, Sleeper LA, Abboud R, Dzavik V, et al. Outcome and profile of ventricular septal rupture with cardiogenic shock after myocardial infarction: a report from the SHOCK Trial Registry. Should we emergently revascularize occluded coronaries in cardiogenic shock? J Am Coll Cardiol. 2000 Sep;36(3 Suppl A):1110-6.

18 Sulzgruber P, El-Hamid F, Koller L, Forster S, Goliasch G, Wojta J, et al. Long-term outcome and risk prediction in patients suffering acute myocardial infarction complicated by postinfarction cardiac rupture. Int J Cardiol. 2017 Jan;227:399-403.

19 Jones BM, Kapadia SR, Smedira NG, Robich M, Tuzcu EM, Menon V, et al. Ventricular septal rupture complicating acute myocardial infarction: a contemporary review. Eur Heart J. 2014 Aug;35(31):2060-8. 